\title{
Identification of a Novel Susceptibility Marker for SARS-CoV-2 Infection in Human Subjects and Risk Mitigation with a Clinically Approved JAK Inhibitor in Human/Mouse Cells
}

Marianne R. Spalinger ${ }^{1,2}$, Rong Hai ${ }^{3}$, Jiang $\mathrm{Li}^{1}$, Alina N. Santos ${ }^{1}$, Tara M. Nordgren ${ }^{1}$, Michel L. Tremblay $^{4}$, Lars Eckmann ${ }^{5}$, Elaine Hanson ${ }^{5}$, Michael Scharl ${ }^{2}$, Xiwei Wu ${ }^{6}$, Brigid S. Boland ${ }^{5}$, Declan F. McCole ${ }^{1}$.

1 Division of Biomedical Sciences, School of Medicine, University of California Riverside, Riverside, California, USA

${ }^{2}$ Department of Gastroenterology and Hepatology, University Hospital Zurich, and University of Zurich, Zurich, Switzerland

${ }^{3}$ Department of Microbiology \& Plant Pathology, University of California Riverside, Riverside, California, USA

${ }^{4}$ Department of Biochemistry and Goodman Cancer Research Centre, McGill University, Montreal, Quebec, Canada

${ }^{5}$ Division of Gastroenterology, University of California San Diego, La Jolla, California, USA

${ }^{6}$ Integrative Genomics Core, Beckman Research Institute of City of Hope, Monrovia, California, USA

${ }^{*}$ Corresponding author: Declan F. McCole, PhD, Division of Biomedical Sciences, School of Medicine, University of California Riverside,

307 School of Medicine Research Building, 900 University Avenue,

Riverside, CA 92521; Tel: (951) 827-7785; E-mail: declan.mccole@ucr.edu

Short title: PTPN2 restricts JAK-STAT induced ACE2 expression in gut and lung epithelium

Author contribution: MRS: study and experiment design, data acquisition, analysis, and interpretation, writing of the manuscript; $\mathrm{RH}, \mathrm{MLT}$ : reagent generation, data analysis and critical intellectual input; TMN: data interpretation and critical intellectual input; ANS, JL: data acquisition, sample collection; LE and EH helped develop the mouse model; BSB provided patient serum samples; MS: collection and analysis of patient samples; DFM: study design, supervision of the experiments, data interpretation, funding, writing of the manuscript. All authors reviewed the manuscript.

Keywords: Coronavirus, COVID-19, SARS-CoV-2, autoimmune disease, genetic susceptibility, Inflammatory Bowel Disease, Tofacitinib 
Funding: This study was supported by the NIH (2R01DK091281, 1R01Al153314 to DFM, K23DK123406 to BSB, and P30DK120515 to LE); a City of Hope-UC Riverside Biomedical Research Initiative (CUBRI) 2016 award (to DFM) and a research stipend from the Swiss National Science Foundation (to MRS). The funding institutions had no role in the study design or in the collection, analysis, and interpretation of data.

Conflict of Interest: BSB consulted for Pfizer Inc, unrelated to the content of this article.

DFM has received investigator-initiated research awards from the makers of Tofacitinib (Pfizer Inc.) through the ASPIRE-Pfizer JAK-STAT in IBD Research Program for studies unrelated to the content of this manuscript. 


\section{ABSTRACT}

Coronavirus disease (COVID-19), caused by SARS-CoV-2, has affected over 65 million individuals and killed over 1.5 million persons (December 8, 2020; www.who.int) ${ }^{1}$. While fatality rates are higher among the elderly and those with underlying comorbidities ${ }^{2}$, host factors that promote susceptibility to SARS-CoV-2 infection and severe disease are poorly understood. Although individuals with certain autoimmune/inflammatory disorders show increased susceptibility to viral infections, there is incomplete knowledge of SARS-CoV-2 susceptibility in these diseases. ${ }^{3-7}$ We report that the autoimmune PTPN2 risk variant rs1893217 promotes expression of the SARS-CoV-2 receptor, ACE2, and increases cellular entry mediated by SARS-CoV-2 spike protein. Elevated ACE2 expression and viral entry were mediated by increased JAK-STAT signalling, and were reversed by the JAK inhibitor, tofacitinib. Collectively, our findings uncover a novel risk biomarker for increased expression of the SARS-CoV-2 receptor and viral entry, and identify a clinically approved therapeutic agent to mitigate this risk.

Despite tremendous effort to understand COVID-19 pathogenesis, risk factors for severe disease are still poorly defined. While most attention has focused on symptoms in the airways, gastrointestinal (GI) symptoms were reported in $46 \%$ of all cases and $33 \%$ presented with GI symptoms in the absence of respiratory symptoms. ${ }^{8,9} \mathrm{Gl}$ symptoms are associated with longer duration and more severe COVID-19 (e.g. increased prevalence of acute renal insufficiency ${ }^{10}$ ), emphasizing their importance for early diagnosis and prognosis. ${ }^{11}$ SARS-CoV-2 can directly infect intestinal epithelial cells, ${ }^{12,13}$ and viral particles have been detected in feces even after virus clearance from the respiratory tract ${ }^{14,15}$. This indicates viral shedding in the gut, which may serve as a reservoir of virus replication, and possible oral-fecal transmission although presence of live virus in feces is disputed. ${ }^{12,16}$

SARS-CoV-2 entry into host cells is mediated by its spike glycoprotein (S protein), which is cleaved by cell surface-associated transmembrane protease serine protease 2 (TMPRSS2) and TMPRSS4 to generate the S1 and S2 subunits in a so-called 'priming' process. ${ }^{16,17} \mathrm{~S} 1$ binds to angiotensin I converting enzyme 2 (ACE2), a monocarboxypeptidase controlling cleavage of several peptides within the renin-angiotensin system. ${ }^{16,17} \mathrm{~S} 2$ drives the subsequent fusion of viral and host membranes. ${ }^{18}$ Interferon (IFN)-JAK-STAT signaling is a suggested major driver of ACE2 expression likely via STAT1/3 binding sites in the ACE2 promoter ${ }^{19}$. ACE2, TMPRSS2, and TMPRSS4 are highly expressed on the surface of epithelial cells such as lung type 2 pneumocytes and absorptive intestinal epithelial cells. ${ }^{12,18-21}$ 
About $16-20 \%$ of the general population carries the single nucleotide polymorphism (SNP) rs1893217 located in the gene locus encoding protein tyrosine phosphatase non-receptor type 2 (PTPN2, also called TCPTP) ${ }^{22,23}$. This SNP causes PTPN2 loss of function and is associated with increased risk for chronic inflammatory and autoimmune diseases including inflammatory bowel disease (IBD), Type 1 diabetes (T1D), and rheumatoid arthritis (RA). ${ }^{24,25}$ PTPN2 directly dephosphorylates several transducers of cytokine receptor signaling including the STAT family of transcription factors (STATs 1/3/5/6)) and Janus kinases (JAK) 1 and JAK 3 that are activated by inflammatory cytokines such as IFN $\gamma .{ }^{26-28} \mathrm{JAK}$ inhibitors have emerged as an effective new therapeutic class in many patients with autoimmune diseases. Indeed a JAK-inhibitor, baricitinib, is in clinical trial (ACTT-2) to reduce disease severity and hospitalization time in COVID-19 ${ }^{29}$. Tofacitinib (Xeljanz ${ }^{\circledR}$ ) is a pan-JAK inhibitor that preferentially inhibits JAK1 and JAK3, is approved to treat RA and the IBD subtype, ulcerative colitis (UC), and we have shown that tofacitinib corrects the consequences of PTPN2-loss in IECs ${ }^{30}$.

Using intestinal samples and peripheral blood mononuclear cells (PBMC) from IBD patients harbouring the autoimmune PTPN2 risk variant rs1893217, human intestinal and lung epithelial cell lines as well as Ptpn2-deficient mouse models, we determined that PTPN2-loss promotes ACE2 expression and entry of viral particles expressing SARS-CoV-2 spikes. Elevated ACE2 expression and viral entry were mediated by increased epithelial JAK-STAT signalling, and were reversed by the clinically-approved JAK inhibitor, Tofacitinib. Collectively, our findings not only describe a risk factor for increased SARS-CoV-2 invasion (entry), but also identify a clinically approved drug that may be utilized to mitigate this risk ${ }^{31}$. 


\section{Results}

\section{PTPN2 regulates ACE2 expression in vivo and in vitro}

Mucosal biopsy samples from IBD patients in the Swiss IBD cohort previously genotyped for the IBD-associated loss-of-function SNP rs1893217 in PTPN2 ${ }^{32}$ were subjected to RNA sequencing. Using the Database for Annotation, Visualization and Integrated Discovery (DAVID), gene ontogeny (GO) biological pathway analysis indicated "Digestion" as the most significantly regulated function based on PTPN2 genotype (presence of risk 'C' allele, patients with the CT or the CC genotype) independent of disease severity (Supplementary Table 1). This biological process included the ACE2 gene, which was also found in three other processes that were increased in ' $C$ ' allele carriers (Supplementary Table 1). Quantitative PCR and Western blotting on intestinal biopsies isolated from Crohn's disease (CD) and ulcerative colitis (UC) patients (Supplementary Table 2) confirmed increased mRNA and protein expression of ACE2 in C-allele carriers (Figure 1A+B). Furthermore ACE2 protein expression negatively correlated with PTPN2 phosphatase activity (Figure 1C). To confirm these findings in Ptpn2-knockout (KO) mice, which exhibit a severe inflammatory phenotype and die within few weeks after birth ${ }^{33}$, we explored ACE2 expression in 3-week-old mice when the intestinal epithelium appears relatively normal compared to heterozygous (Het) and wild-type (WT) littermates. Although Ptpn2-KO mice did not exhibit any difference in Ace2 mRNA expression in whole intestinal samples compared with wild-type (WT) mice (Supplementary Figure 1A), Ace2 mRNA and protein expression in intestinal epithelial cells (IEC) from these mice was significantly increased (Supplementary Figure 1A, Figure 1D). In addition, Ace2 mRNA expression in lung and cardiac tissue was significantly increased in Ptpn2-KO mice (Figure 1D, Supplementary Figure 1B+C). Given the strong increase of ACE2 expression in IECs of Ptpn2-KO mice, and to explore whether PTPN2 regulates ACE2 in the absence of inflammation, we confirmed these findings in mice lacking PTPN2 specifically in IECs (Ptpn $2^{\Delta \mathrm{IEC}}$ mice). Also in these mice, Ace2 mRNA and protein expression were clearly elevated in PTPN2-deficient IECs (Figure 1E, Supplementary Figure 1D), demonstrating that the increase in Ace2 expression was not dependent on inflammation. This effect was confirmed in intestinal epithelial, lung epithelial and monocyte cell lines upon PTPN2 knockdown, where depletion of PTPN2 resulted in elevated ACE2 expression (Supplementary Figure 1E). ${ }^{28}$ Notably, the serine proteases TMPRSS2 and TMPRSS4, which are additional cofactors of SARS-CoV-2 viral entry, were not altered in PTPN2 knockdown (PTPN2-KD) cells (Supplementary Figure 2). This suggests that PTPN2 specifically regulates ACE2 expression. 
PTPN2 negatively regulates SARS-CoV-2 spike protein-mediated viral entry into epithelial and immune cells

To assess the functional consequence of increased ACE2 expression in PTPN2-deficient cells, we assessed whether deletion of PTPN2 affects the uptake of virus-like particles (VLPs) expressing SARS-CoV-2 spikes. Apical uptake of empty VLPs (-), which served as a negative control, or uptake of VLPs covered with spike G glycoprotein of the rhabdovirus vesicular stomatitis virus $(G)$, which served as a positive control for viral entry, was not affected in PTPN2-KD Caco-2BBe (Figure 2A) and HT-29.cl19A IECs (Figure 2B) or A549 lung epithelial cells (Figure $2 \mathrm{C}$ ), thus indicating that non-specific uptake was not affected upon PTPN2 knockdown. In contrast, VLPs expressing SARS CoV-2 spikes entered PTPN2-KD epithelial cells more efficiently than control cells (Figure 2A-C), indicating that PTPN2 deficiency not only promotes ACE2 expression but also viral uptake into intestinal and lung epithelial cells. Notably, loss of PTPN2 also caused a significant increase in ACE2 expression in PTPN2-KD monocytes (Supplementary Figure 1) and increased CoVS entry (Figure 2D). This indicates that PTPN2 regulation of ACE2 and SARS-CoV-2 spike-expressing VLP entry is not restricted to epithelial cells but has similar functional consequences in immune cells. Furthermore, increased uptake of SARS-CoV-2 spike-expressing VLPs in PTPN2-deficient cells was no longer observed upon inhibition of ACE2 with a blocking antibody (Supplementary Figure 3), indicating that ACE2 mediated SARS-CoV-2 spike-expressing VLP uptake. In summary, this indicates that loss of PTPN2 promotes SARS-CoV-2 uptake by promoting ACE2 expression.

\section{IFN- $\gamma$ promotes ACE2 expression and SARS-CoV-2 Spike protein uptake}

It has been suggested that ACE2 expression is induced by interferons ${ }^{34}$ and its promoter is reported to have putative STAT1 binding sites ${ }^{19,35}$, although newer findings debate whether interferons can induce ACE2 expression, or if it drives expression and release of a shorter version of the protein $^{36}$. Since PTPN2 is a potent suppressor of IFN- $\gamma$-induced signaling cascades $^{27,37}$, and directly dephosphorylates STAT1 ${ }^{38}$, we assessed whether IFN- $\gamma$ treatment promotes ACE2 expression in our cell culture models. Indeed, IFN- $\gamma$ promoted ACE2 expression and this was further increased in PTPN2-KD cells (Figure 3A, Supplementary Figure 4). Silencing of STAT1 using siRNA constructs prevented IFN- $\gamma$-induced ACE2 mRNA and protein expression and STAT1-siRNA treated PTPN2-KD Caco-2BBe, A549 and THP-1 cells expressed ACE2 levels comparable to those in Ctr cells (Figure 3B+C and Supplementary Figure 5 ). In line with these effects on ACE2 expression, uptake of SARS-CoV-2 S protein-expressing VLPs was elevated in IFN- $\gamma$-treated control and PTPN2-KD cells, while STAT1 silencing normalized the 
uptake, both in Ctr cells and PTPN2-KD cells (Figure 3D+E, Supplementary Figure 5). This strongly suggests that deletion of PTPN2 promotes ACE2 expression and SARS-CoV-2 entry in a STAT1-dependent manner and inhibition of STAT signaling may mitigate elevated ACE2 expression and SARS-CoV-2 entry into host cells.

\section{Tofacitnib reverses ACE2 overexpression and increased SARS-CoV-2 entry in PTPN2- deficient human cells and mice}

To test whether inhibition of STAT activation can indeed prevent elevated ACE2 expression in PTPN2-deficient cells, we next treated Caco-2BBe IECs with the pan-JAK-inhibitor tofacitinib. Similar to our findings in cells treated with STAT1 siRNA, inhibition of JAK-STAT signalling in Caco-2BBe cells using tofacitinib prevented IFN- $\gamma$-induced increases in ACE2 mRNA and protein expression and normalized the elevated ACE2 levels in PTPN2-KD cells (Figure 4A+B). Moreover, tofacitinib reduced ACE2-mediated SARS-CoV-2 spike-expressing VLP uptake in both PTPN2-KD and in IFN- $\gamma$-treated cells (Figure 4C). Similar effects were observed in A549 lung epithelial cells (Supplementary Figure 6), indicating that tofacitinib treatment might not only reduce ACE2-mediated intestinal viral uptake, but also reduce viral uptake in the respiratory tract, the primary entry site of SARS-CoV-2. We next tested if tofacitinib altered ACE2 levels in human subjects. Levels of soluble ACE2, which has been suggested to reduce viral binding to host cells ${ }^{39}$, were not altered in UC patients treated with tofacitinib when compared to UC patients under anti-TNF treatment with similar disease activity (Figure 4D, Supplementary Table $3)$. This suggests that tofacitinib treatment does not reduce shedding/release of ACE2 into serum. In contrast, when analysing ACE2 levels in PBMCs from IBD patients carrying the PTPN2 loss-of-function SNP rs1893217, we again observed that ACE2 mRNA and protein levels and SARS-CoV-2 spike-expressing VLP entry were clearly elevated in variant carriers compared to non-carriers (Figure 4E-G). Notably, treatment with tofacitinib not only reduced ACE2 levels and viral entry in variant carriers, but also in non-carriers (Figure 4E-G). These findings indicate that loss of PTPN2 or presence of the loss-of-function variant in PTPN2 promotes ACE2 expression and subsequently facilitates uptake of SARS-CoV-2-spikeexpressing VLPs, and that treatment with tofacitinib can mitigate this potential risk by reducing ACE2 cellular expression rather than affecting release of soluble ACE2. Furthermore, our data strongly indicate that treatment with tofacitinib might not only be beneficial in PTPN2 variant carriers, but also for non-carriers. 
Our data consistently demonstrate that PTPN2 dysfunction promotes expression of ACE2 and uptake of SARS-CoV-2 spike protein, and this is further increased by inflammation. This is striking given a recent paper identifying that non-genotyped IBD patients (+/- inflammation) showed no change in ACE2 or TMPRSS2 expression, while experimental colitis in mice reduced gut epithelial Ace 2 expression. ${ }^{40}$ This indicates that inflammation does not promote ACE2 expression per se, but that the elevated ACE2 levels in patients or mice with reduced PTPN2 activity are indeed due to PTPN2 deficiency.

Summarized, we demonstrate that SNP rs1893217 in PTPN2 is associated with increased expression of ACE2 and SARS-CoV-2 entry, and potentially represents one of the first identified COVID-19 genetic susceptibility biomarkers. By using samples collected well before the COVID19 outbreak, our identification of a genetic susceptibility marker avoids the potential for ascertainment bias in most genetic studies of COVID-19, as clinically significant COVID-19 patients are more likely to be included in research projects than asymptomatic cases ${ }^{41}$. With respect to genetic markers of COVID-19 susceptibility, studies have proposed the involvement of $A B O$ blood groups, with blood group $O$ associated with lower risk, while blood group $A$ was associated with higher risk of acquiring COVID-19 compared with non-A blood groups ${ }^{41-44}$. However, this correlation did not culminate in therapeutic implications. Moreover, a cluster of genes on chromosome 3 has been linked with increased severity, although this may have distinct geographic distributions ${ }^{41,45}$. In contrast, our finding of increased ACE2 expression/viral particle uptake in PTPN2 variant cells might not only indicate a potentially novel genetic marker for increased disease, but also identifies tofacitinib - a drug already approved for treatment of arthritis and IBD - and potentially other JAK inhibitors such as baricitinib, as a potential therapeutic strategy to specifically mitigate this risk. 


\section{Methods}

Patient samples. Samples from IBD patients used for RNA and protein isolation were obtained from the Swiss IBD cohort and the sample use approved by the local ethic's board (Ethic's board of the Kanton Zurich, Switzerland; approval number EK1977). Serum samples were obtained from University of California San Diego under IRB Protocol \# 131487. All patients provided informed consent.

Mice. PTPN2-knock-out (KO) mice in the BALB/c background were a gift from Prof. M. Tremblay at McGill University in Montreal. To generate mice lacking PTPN2 specifically in intestinal epithelial cells ( $\triangle \mathrm{IEC}$ mice), mice with a loxP-flanked exon 3 of the PTPN2 gene (PTPN2-fl/fl mice, originally obtained from EUCOMM, abbreviated as $\mathrm{fl} / \mathrm{fl}$ ) were crossed with VillinCre-ERT2 mice (Jackson Laboratories). Translocation of the Cre-ERT2 construct and subsequent recombination and deletion of the floxed gene was induced by tamoxifen-injections (i.p., $1 \mathrm{mg} / \mathrm{mouse} /$ day) on 5 consecutive days. All mouse experiments were conducted according to protocols approved by the IACUC commission of the University of California Riverside (AUP20190032).

Protein isolation and Western blotting. Protein isolation and Western blotting were performed according to standard procedures. For protein isolation from cells, the cells were washed with ice cold PBS and lysed in RIPA buffer containing phosphatase and protease inhibitors (Roche, South San Francisco, CA). For mouse and human biopsies, the samples were dissociated in RIPA buffer using a bead-beater and metal beads. All samples were then sonicated for 30 seconds, centrifuged (10 min. at $12^{\prime} 000 \mathrm{~g}$ at $\left.4^{\circ} \mathrm{C}\right)$, and the supernatant transferred into fresh tubes. Protein concentration was detected using a BCA assay (Thermo Fisher Scientific, Waltham, MA). For Western blots, aliquots with equal amounts of protein were separated by electrophorese on polyacrylamide gels, and the proteins blotted on nitrocellulose membranes. The membranes were then blocked in blocking buffer (3\% milk, 1\% BSA in tris-buffered saline with $0.5 \%$ Tween) for $1 \mathrm{~h}$ and incubated over night at $4^{\circ} \mathrm{C}$ with anti-ACE2 (Clone E-11, Santa Cruz Biotechnology), anti-phospho-STAT1 (Tyr701, clone 58D6; Cell Signalling Technologies, Danvers, MA), anti-STAT1 (clone 42H3; Cell Signalling Technologies), or anti- $\beta$-actin (Clone AC-74, Sigma-Aldrich, St. Louis, MO) antibodies. On the next day, the membranes were washed in tris-buffered saline with $0.5 \%$ Tween, incubated with HRP-coupled secondary antibodies (Jackson Immunolabs, West Grove, PA), washed again, and immunoreactive 
proteins detected using ELC substrate (Thermo Fisher Scientific) and X-ray films (GE Healthcare, Chicago, IL).

PTPN2 phosphatase assay. For PTPN2 activity measurements, $100 \mu \mathrm{g}$ protein lysates were pre-cleared for $1 \mathrm{~h}$ using Sepharose A beads, incubated with $2 \mu \mathrm{l}$ anti-PTPN2 antibody (Clone D7T7D, Cell Signaling Technologies) over night, incubated with Sepharose A beads for $1 \mathrm{~h}$ and centrifuged ( $3 \mathrm{~min}$. at $12^{\prime} 000 \mathrm{~g}$ at $4^{\circ} \mathrm{C}$ ). The precipitates were washed 3 times with ice cold PBS and the beads resuspended in phosphatase assay buffer (Thermo Fisher Scientific) and phosphatase activity measured using the EnzCheck Phosphatase assay (Thermo Fisher Scientific) according to the manufacturer's instructions.

RNA isolation and qPCR. For RNA isolation, biopsies were disrupted in RLT buffer (Qiagen, Valencia, CA) using a bead beater and metal beads. Cells were washed twice in ice-cold PBS before lysis in RLT buffer. All samples were then passed 3-5 times through a 26G needle prior to RNA isolation using the RNAeasy mini kit from Qiagen. RNA concentrations were estimated by absorbance measurement at 260 and $280 \mathrm{~nm}$, and cDNA generated using the qScript reverse transcriptase (Quantabio, Beverly MA). Quantitative real-time PCR was performed using iQ SYBR Green Supermix (BioRad, Hercules, CA) on a C1000 Thermal cycler equipped with a CFX96 Real-Time PCR system using BioRad CFX Manager 3.1 Software. Measurements were performed in triplicates using GAPDH as an endogenous control. Results were analyzed by the $\triangle \triangle C T$ method. The real-time PCR included an initial enzyme activation step (3 minutes, $\left.95^{\circ} \mathrm{C}\right)$ followed by 45 cycles consisting of a denaturing $\left(95^{\circ} \mathrm{C}, 10\right.$ seconds), an annealing $\left(53^{\circ}\right.$ $60^{\circ} \mathrm{C}, 10$ seconds) and an extending $\left(72{ }^{\circ} \mathrm{C}, 10\right.$ seconds) step. The used primers are listed in Supplementary Table S3.

VLPs and measurement of VLP uptake. To produce pseudoparticles, 293T cells were transfected with plasmids encoding a minimal HIV (pTRIP, CSGW, CSPW) provirus expressing the Gaussia Luciferase (Gluc), gag-pol, and $S$ protein of SARS-CoV-2 virus using polyethylenimine (PEI) transfection reagent ${ }^{46-48}$. Supernatants were collected at 24,48 and 72 hours post-transfection, pooled, filtered $(0.45 \mathrm{~mm})$, aliquoted and stored at $-80^{\circ} \mathrm{C}$. Pseudoparticle infections were performed in the presence of $4 \mu \mathrm{g} / \mathrm{ml}$ polybrene. Appropriate amounts of pseudoparticle were added onto target cells and plates incubated for $3 \mathrm{hrs}\left(37^{\circ} \mathrm{C}\right)$ before changing media. Gaussian luciferase was measured at 24,48 , and 72 hrs after infection. 
To measure VLP uptake into cells, the VLP-containing medium was diluted 1:2 in cell culture medium and applied to the cells. After $1 \mathrm{~h}$, the cells were washed with PBS and fresh medium added to the cells. In experiments with IFN- $\gamma(1000 \mathrm{IU} / \mathrm{ml}$; Roche, Belmont CA), the replacement medium for cells infected in presence of IFN- $\gamma$ contained IFN- $\gamma$ as well. To determine VLP uptake, luciferase luminescence in cell culture supernatant was determined using the Renilla luciferase activity assay from Thermo Fisher Scientific.

Cell culture, PTPN2 knockdown, siRNA treatment and IFN- $\gamma$ treatment. HT-29.cl19A were obtained from Kim E. Barrett (University of California, San Diego, California), Caco-2BBe, A549 and THP-1 cells were originally obtained from ATCC and cultured according to the manufacturer's recommendation in medium with 10\% FCS. For PTPN2 knockdown, the cells were infected with lentiviral particles containing non-targeting control shRNA (Ctr) or PTPN2specfic shRNA as described previously ${ }^{49}$ and stable clones selected using puromycin. For STAT1 silencing, the cells were transfected with previously validated, STAT1-specific or nontargeting control siRNA constructs (Dharmacon) using DharmaFECT transfectionre agents as described previously ${ }^{50}$. In experiments with STAT1 siRNA and IFN- $\gamma$ treatment, the culture medium was replaced with serum-free medium $8 \mathrm{~h}$ prior to addition of IFN- $\gamma$ (1000 IU; Roche). In experiments with Tofacitinib, the cells were treated with tofacitinib (50 $\mu \mathrm{M}$, MedChemExpress, Monmouth Junction, $\mathrm{NJ}$ ). Control cells were treated with an equal amount of vehicle (dimethyl sulfoxide, DMSO, 0.5\%, Sigma-Aldrich).

ELISA. Human ACE2 ELISA was obtained from R\&D and performed according to the manufacturer's instructions with undiluted serum.

RNA sequencing. RNA sequencing was performed and analyzed by the Integrative Genomics Core, City of Hope National Medical Center (Duarte USA). The RNA-seq libraries were constructed with Kapa mRNA HyperPrep Kit (Roche) following the manufacturer's recommendation. The libraries were then sequenced on an Illumina Hiseq 2500 with single end $50 \mathrm{bp}$ reads to a depth of about $35 \mathrm{M}$. The sequences were aligned to human genome assembly hg38 using Tophat2 v2.0.14. RNA-seq data quality was evaluated using RSeQC v2.5. For each sample, expression counts for Ensembl genes (v92) were summarized by HTseq v0.6.1, and reads per kilobase of transcript per million mapped reads (RPKM) were calculated. Count normalization and differential expression analyses between groups were conducted using Bioconductor package "DESeq2" v1.14.1. Heatmaps were generated using R package 
"heatmap3". The Gene Ontology and pathway analysis was performed using DAVID online tools and Ingenuity Pathway Analysis (IPA).

Statistics. Data are represented as mean of a series of $n$ biological repetitions \pm standard deviation (SD). Data followed a Gaussian distribution and variation was similar between groups for conditions analyzed together. Significant differences were determined using GraphPad Prism v9 software using analysis of variance (ANOVA). p-values below 0.05 were considered significant. Mice for ex vivo analyses were matched for age and sex. Numbers of replicates are given in the figure legends. No data points were excluded from statistical analysis. 


\section{Author contribution}

MRS: data acquisition, analysis and interpretation, study design, writing the manuscript; JL, ANS: data acquisition; $\mathrm{RH}, \mathrm{MLT}$ : generation of material for the study and technical input; $\mathrm{RH}$, TMN: critical intellectual input; XW: sequencing and bioinformatics; LE, EH, BSB, MS: human sample acquisition; DFM: conceived the study, data interpretation, manuscript writing, acquisition of funding.

\section{Competing interest statement}

BSB consulted for Pfizer Inc, unrelated to the content of this article. DFM has received investigator-initiated research awards from the makers of Tofacitinib (Pfizer Inc.) through the ASPIRE-Pfizer JAK-STAT in IBD Research Program for studies unrelated to the content of this manuscript.

\section{Additional Information}

Supplementary Information is available for this paper.

Correspondence and requests for materials should be addressed to Declan F. McCole. 


\section{References}

1. Wu, F., et al. A new coronavirus associated with human respiratory disease in China (vol 579, pg 265, 2020). Nature 580, E7-E7 (2020).

2. McArthur, L., et al. Review of Burden, Clinical Definitions, and Management of COVID-19 Cases. The American Journal of Tropical Medicine and Hygiene 103, 625-638 (2020).

3. Favalli, E.G., et al. COVID-19 infection and rheumatoid arthritis: Faraway, so close! Autoimmun Rev 19, 102523 (2020).

4. Control, C.f.D. Preliminary Estimates of the Prevalence of Selected Underlying Health Conditions Among Patients with Coronavirus Disease 2019 - United States, February 12-March 28, 2020. MMWR Morb Mortal Wkly Rep (2020).

5. Barron, E., et al. Associations of type 1 and type 2 diabetes with COVID-19-related mortality in England: a whole-population study. The lancet. Diabetes \& endocrinology 8, 813-822 (2020).

6. Lukin, D.J., et al. Baseline Disease Activity and Steroid Therapy Stratify Risk of COVID-19 in Patients With Inflammatory Bowel Disease. Gastroenterology 159, 1541-1544.e1542 (2020).

7. Wisniewski, A., et al. Increased incidence of systemic serious viral infections in patients with inflammatory bowel disease associates with active disease and use of thiopurines. United European Gastroenterol J 8, 303-313 (2020).

8. Pan, L., et al. Clinical Characteristics of COVID-19 Patients With Digestive Symptoms in Hubei, China: A Descriptive, Cross-Sectional, Multicenter Study. The American journal of gastroenterology 115, 766-773 (2020).

9. Gao, Q.Y., Chen, Y.X. \& Fang, J.Y. 2019 Novel coronavirus infection and gastrointestinal tract. J Dig Dis 21, 125-126 (2020).

10. Cholankeril, G., et al. Association of Digestive Symptoms and Hospitalization in Patients With SARS-CoV-2 Infection. Am. J. Gastroenterol. 115, 1129-1132 (2020).

11. Gu, J., Han, B. \& Wang, J. COVID-19: Gastrointestinal Manifestations and Potential Fecal-Oral Transmission. Gastroenterology 158, 1518-1519 (2020).

12. Zang, R., et al. TMPRSS2 and TMPRSS4 promote SARS-CoV-2 infection of human small intestinal enterocytes. Sci Immunol 5(2020).

13. Lamers, M.M., et al. SARS-CoV-2 productively infects human gut enterocytes. Science (2020).

14. Ma, C.X., Cong, Y.Z. \& Zhang, H. COVID-19 and the Digestive System. Am. J. Gastroenterol. 115, 1003-1006 (2020).

15. Ye, Q., Wang, B., Zhang, T., Xu, J. \& Shang, S. The mechanism and treatment of gastrointestinal symptoms in patients with COVID-19. American Journal of Physiology-Gastrointestinal and Liver Physiology 319, G245-G252 (2020).

16. Neurath, M.F. Covid-19 and immunomodulation in IBD. Gut 0, 1-8 (2020).

17. Yan, R., et al. Structural basis for the recognition of SARS-CoV-2 by full-length human ACE2. Science 367, 1444-1448 (2020).

18. Hoffmann, M., et al. SARS-CoV-2 Cell Entry Depends on ACE2 and TMPRSS2 and Is Blocked by a Clinically Proven Protease Inhibitor. Cell 181, 271-280 e278 (2020).

19. Ziegler, C.G.K., et al. SARS-CoV-2 receptor ACE2 is an interferon-stimulated gene in human airway epithelial cells and is detected in specific cell subsets across tissues. Cell 181, 1-20 (2020).

20. Xiao, F., et al. Evidence for Gastrointestinal Infection of SARS-CoV-2. Gastroenterology 158, 1831-1833 e1833 (2020).

21. Hamming, I., et al. Tissue distribution of ACE2 protein, the functional receptor for SARS coronavirus. A first step in understanding SARS pathogenesis. The Journal of pathology 203, 631637 (2004). 
22. Todd, J.A., et al. Robust associations of four new chromosome regions from genome-wide analyses of type 1 diabetes. Nat. Genet. 39, 857-864 (2007).

23. Spalinger, M.R., et al. The Clinical Relevance of the IBD-Associated Variation within the Risk Gene Locus Encoding Protein Tyrosine Phosphatase Non-Receptor Type 2 in Patients of the Swiss IBD Cohort. Digestion 93, 182-192 (2016).

24. Spalinger, M.R., et al. The Clinical Relevance of the IBD-Associated Variation within the Risk Gene Locus Encoding Protein Tyrosine Phosphatase Non-Receptor Type 2 in Patients of the Swiss IBD Cohort. Digestion 93, 182-192 (2016).

25. Scharl, M., et al. Crohn's disease-associated polymorphism within the PTPN2 gene affects muramyl-dipeptide-induced cytokine secretion and autophagy. Inflamm. Bowel Dis. 18, 900-912 (2012).

26. Simoncic, P.D., Lee-Loy, A., Barber, D.L., Tremblay, M.L. \& McGlade, C.J. The T cell protein tyrosine phosphatase is a negative regulator of janus family kinases 1 and 3. Curr. Biol. 12, 446453 (2002).

27. Scharl, M., et al. Protection of epithelial barrier function by the Crohn's disease associated gene protein tyrosine phosphatase n2. Gastroenterology 137, 2030-2040 e2035 (2009).

28. Krishnan, M. \& McCole, D.F. T cell protein tyrosine phosphatase prevents STAT1 induction of claudin-2 expression in intestinal epithelial cells. Ann. N. Y. Acad. Sci. 1405, 116-130 (2017).

29. ClinicalTrials.gov. Adaptive COVID-19 Treatment Trial 2 (ACTT-2). Vol. 2020 Clinical Trial (ClinicalTrials.gov, https://clinicaltrials.gov/ct2/show/NCT04401579, 2020).

30. Spalinger, M.R., et al. The JAK inhibitor tofacitinib rescues intestinal barrier defects caused by disrupted epithelial-macrophage interactions. J Crohns Colitis (2020).

31. La Rosée, F., et al. The Janus kinase 1/2 inhibitor ruxolitinib in COVID-19 with severe systemic hyperinflammation. Leukemia 34, 1805-1815 (2020).

32. Yilmaz, B., et al. The presence of genetic risk variants within PTPN2 and PTPN22 is associated with intestinal microbiota alterations in Swiss IBD cohort patients. PLoS One 13, e0199664 (2018).

33. You-Ten, K.E., et al. Impaired bone marrow microenvironment and immune function in T cell protein tyrosine phosphatase-deficient mice. The Journal of experimental medicine 186, 683-693 (1997).

34. Ziegler, C.G.K., et al. SARS-CoV-2 Receptor ACE2 Is an Interferon-Stimulated Gene in Human Airway Epithelial Cells and Is Detected in Specific Cell Subsets across Tissues. Cell 181, 1016-+ (2020).

35. Barker, H. \& Parkkila, S. Bioinformatic characterization of angiotensin-converting enzyme 2, the entry receptor for SARS-CoV-2. PLoS One 15, e0240647 (2020).

36. Saffern, M. ACE2 is not induced by interferon. Nature Reviews Immunology 20, 521-521 (2020).

37. Scharl, M., Hruz, P. \& McCole, D.F. Protein tyrosine phosphatase non-receptor Type 2 regulates IFN-gamma-induced cytokine signaling in THP-1 monocytes. Inflamm. Bowel Dis. 16, 2055-2064 (2010).

38. ten Hoeve, J., et al. Identification of a nuclear Stat1 protein tyrosine phosphatase. Mol. Cell. Biol. 22, 5662-5668 (2002).

39. Ciaglia, E., Vecchione, C. \& Puca, A.A. COVID-19 Infection and Circulating ACE2 Levels: Protective Role in Women and Children. Frontiers in pediatrics 8, 206 (2020).

40. Burgueno, J.F., et al. Expression of SARS-CoV-2 Entry Molecules ACE2 and TMPRSS2 in the Gut of Patients With IBD. Inflammatory bowel diseases 26, 797-808 (2020).

41. Genomewide Association Study of Severe Covid-19 with Respiratory Failure. N. Engl. J. Med. 383, 1522-1534 (2020). 
42. Wu, B.B., Gu, D.Z., Yu, J.N., Yang, J. \& Shen, W.Q. Association between ABO blood groups and COVID-19 infection, severity and demise: A systematic review and meta-analysis. Infect. Genet. Evol. 84, 104485 (2020).

43. Zietz, M., Zucker, J. \& Tatonetti, N.P. Testing the association between blood type and COVID-19 infection, intubation, and death. medRxiv (2020).

44. Zhao, J., et al. Relationship between the ABO Blood Group and the COVID-19 Susceptibility. Clin. Infect. Dis. (2020).

45. Zeberg, H. \& Pääbo, S. The major genetic risk factor for severe COVID-19 is inherited from Neanderthals. Nature (2020).

46. Ploss, A., et al. Human occludin is a hepatitis $C$ virus entry factor required for infection of mouse cells. Nature 457, 882-886 (2009).

47. Pica, N., et al. Hemagglutinin stalk antibodies elicited by the 2009 pandemic influenza virus as a mechanism for the extinction of seasonal H1N1 viruses. Proc. Natl. Acad. Sci. U. S. A. 109, 25732578 (2012).

48. Hai, R., et al. Influenza viruses expressing chimeric hemagglutinins: globular head and stalk domains derived from different subtypes. J. Virol. 86, 5774-5781 (2012).

49. Spalinger, M.R., et al. PTPN2 Regulates Interactions Between Macrophages and Intestinal Epithelial Cells to Promote Intestinal Barrier Function. Gastroenterology (2020).

50. Sayoc-Becerra, A., et al. The JAK-Inhibitor Tofacitinib Rescues Human Intestinal Epithelial Cells and Colonoids from Cytokine-Induced Barrier Dysfunction. Inflamm. Bowel Dis. 26, 407-422 (2020). 
A

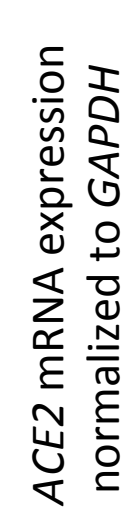

B

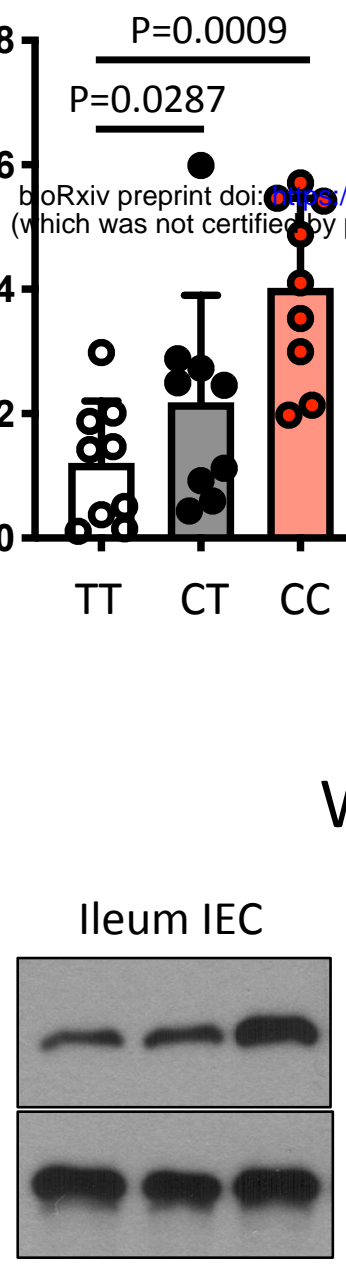

WT Het $\mathrm{KO}$

Whole Body KO

D

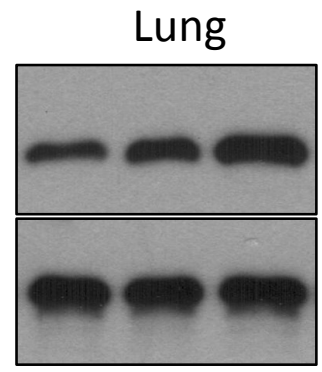

WT Het $\mathrm{KO}$

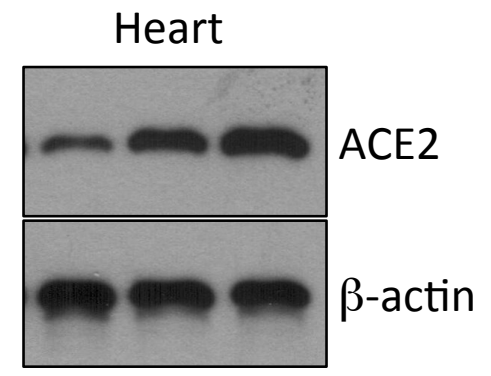

WT Het $\mathrm{KO}$
C

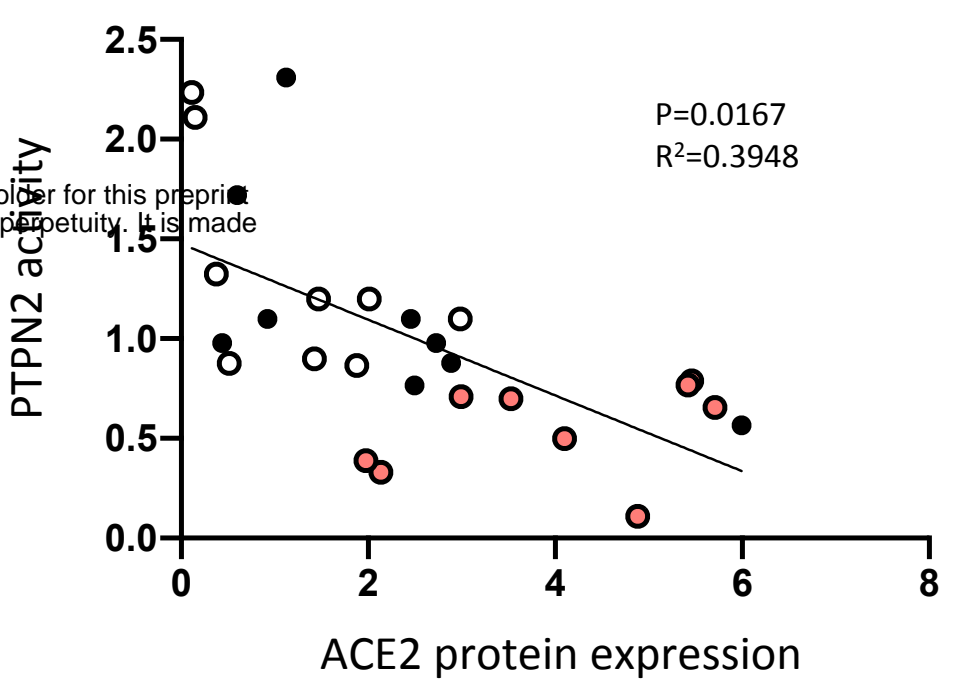

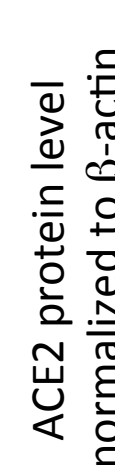

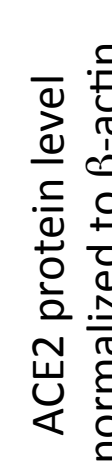

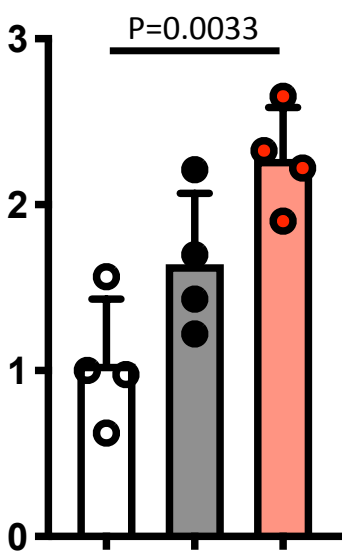

WT Het $\mathrm{KO}$

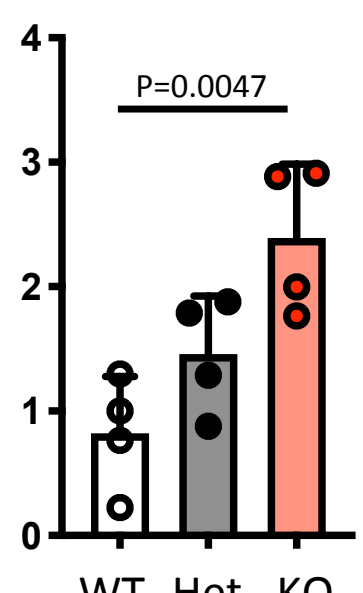

WT Het KO

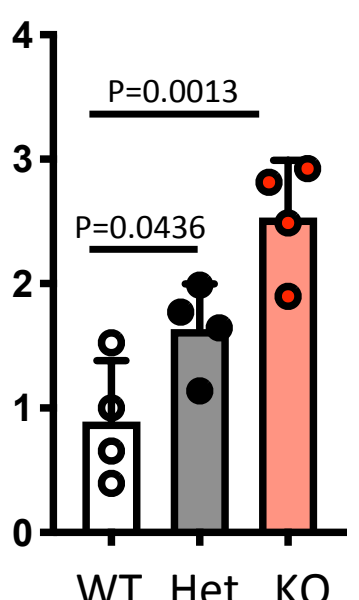

$E$

Ptpn $2^{\mathrm{AIEC}}$

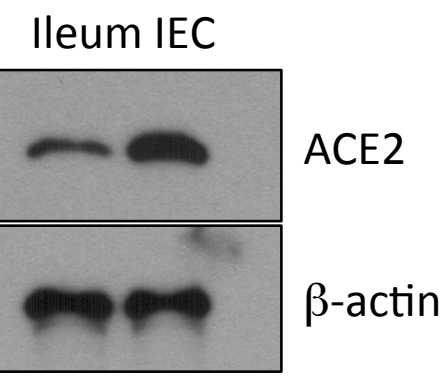

fl/fl $\Delta \mathrm{IEC}$

Figure 1. Presence of SNP rs1893217 in PTPN2 promotes ACE2 expression. A) lleum and colon biopsies from IBD patients homozygous for the major allele (TT), heterozygous (CT) or homozygous for the inflammatory disease-associated minor allele (CC) in PTPN2 SNP rs1893217 were analyzed for A) ACE2 mRNA and B) ACE2 protein expression. Depicted are representative Western blot pictures and values below the blot indicate relative band density normalized to $\beta$-actin and TT controls. C) PTPN2 phosphatase activity levels were analyzed in the same samples as in B and correlated with relative PTPN2 protein levels. D+E) Representative Western blot pictures and respective densitometric analysis for ACE2 and $\beta$-actin in intestinal epithelial cells (IEC) from the illeum, lung tissue, and heart tissue from 3-week-old wild-type (WT), whole-body Ptpn2 heterozygous (Het) or Ptpn2 knock-out (KO) mice (D) or mice in which Ptpn2 was specifically deleted in IECs $(\Delta \mathrm{IEC})$ or their control littermates $(\mathrm{fl} / \mathrm{fl})(\mathrm{E})$. Statistical differences are indicated in the figures (One-way ANOVA $(A+B, D+E)$ or linear regression $(C)), A-C: n=8$ per genotype, $D+E: n=4$. Each dot represents a biological replicate. 
A

IEC cell lines

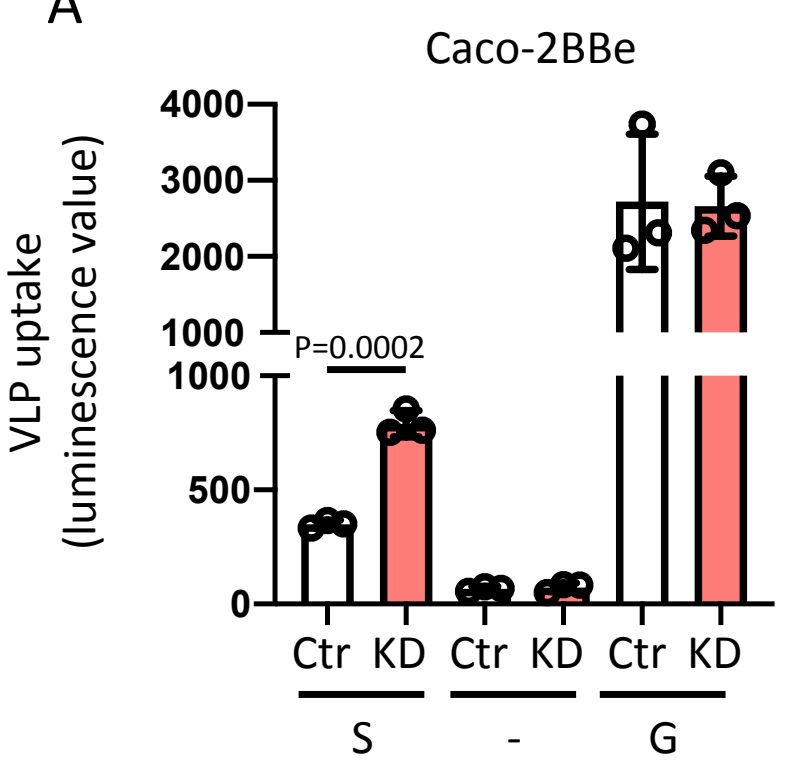

B Lung epithelial cell line

A549

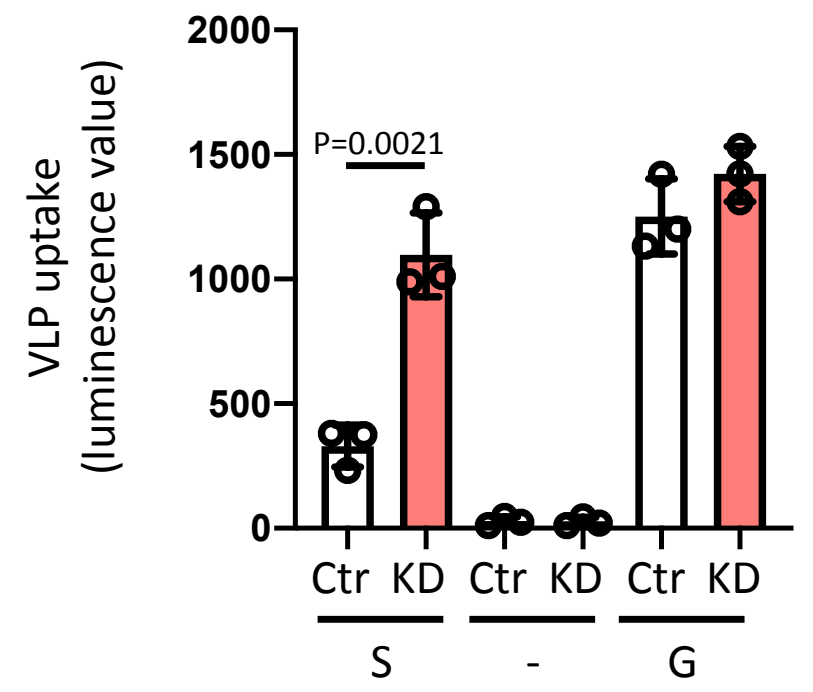

HT29.cl19A

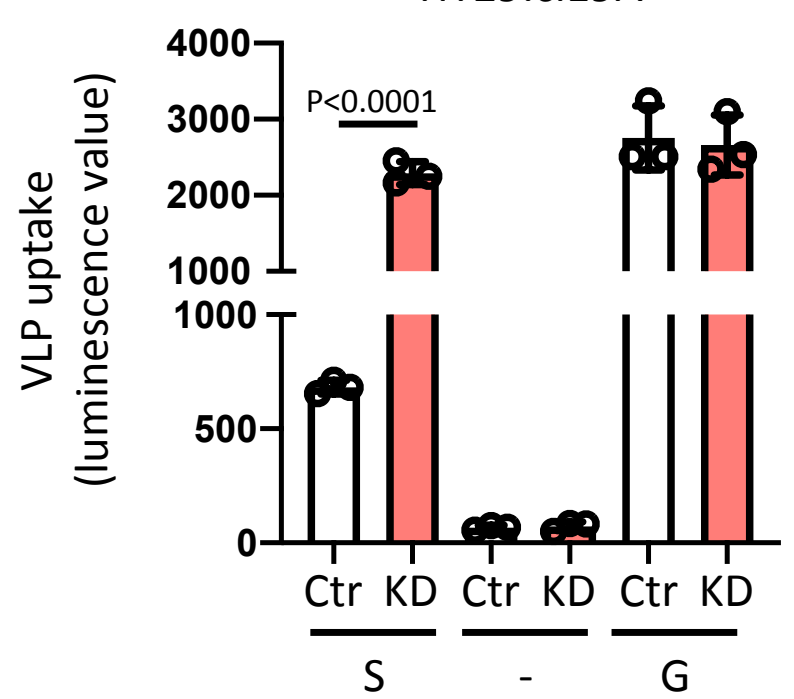

C Monocytic cell line

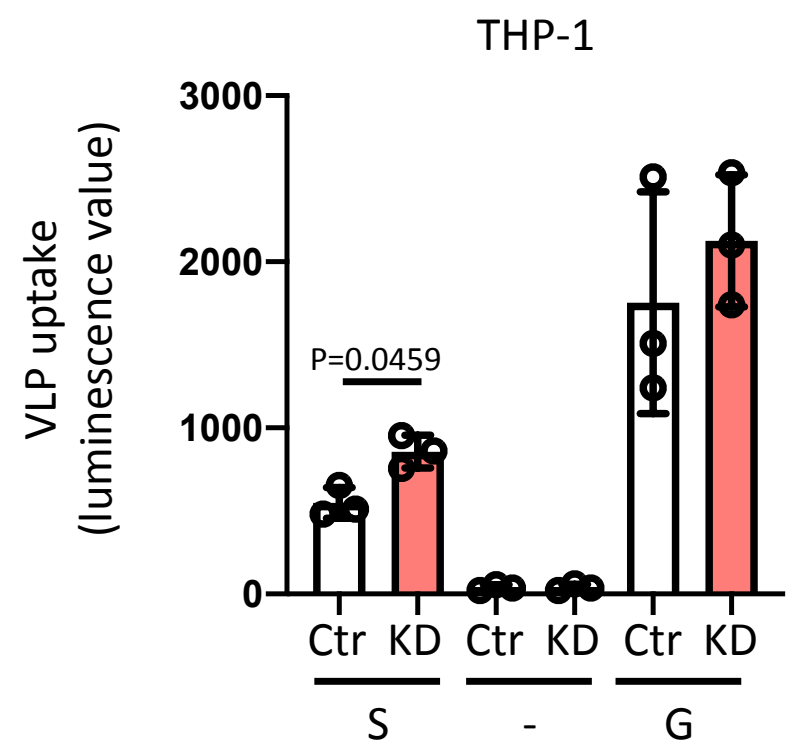

Figure 2. PTPN2 knockdown facilitates entry of VLPs expressing SARSCoV-2 spike S protein. A) Caco-2BBe, B) HT-29.cl19A, C) A549, and D) THP-1 cells expressing non-targeting control (Ctr) or PTPN2-specific (KD) shRNA were incubated with virus like particles (VLPs) expressing renilla luciferase and SARS-CoV-2 spike protein (S), no additional proteins (-, negative control), or the spike $G$ glycoprotein of the rhabdovirus vesicular stomatitis virus ( $G$, positive control). $48 \mathrm{~h}$ after infection, luminescence values of the supernatant were measured as an approximation of VLP uptake. Statistical differences are indicated in the figure (Student's $t$ test, $n=3$ independent experiments). Each dot represents the average of an independent experiment with 2-3 technical replicates, each. 
A

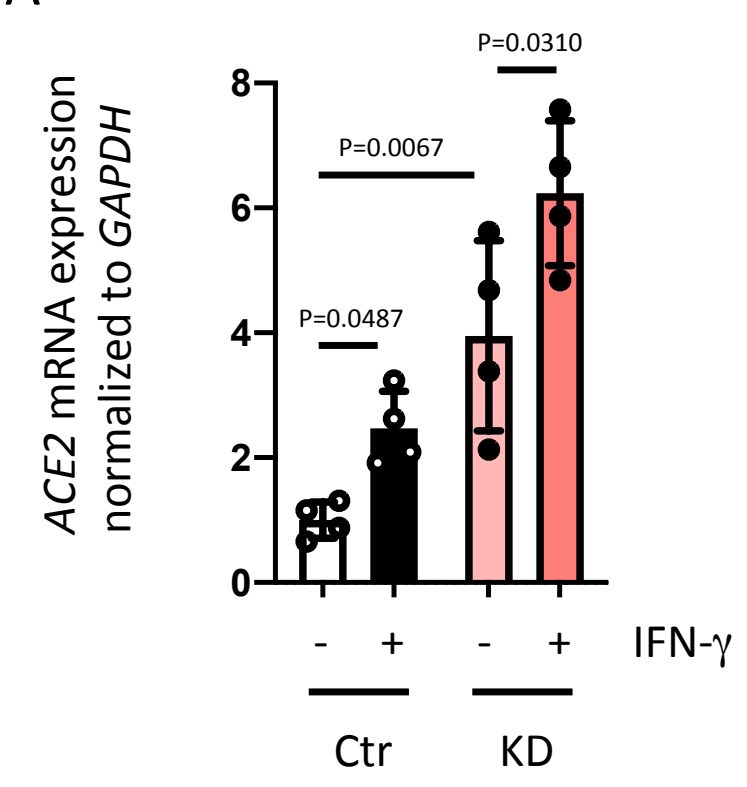

D

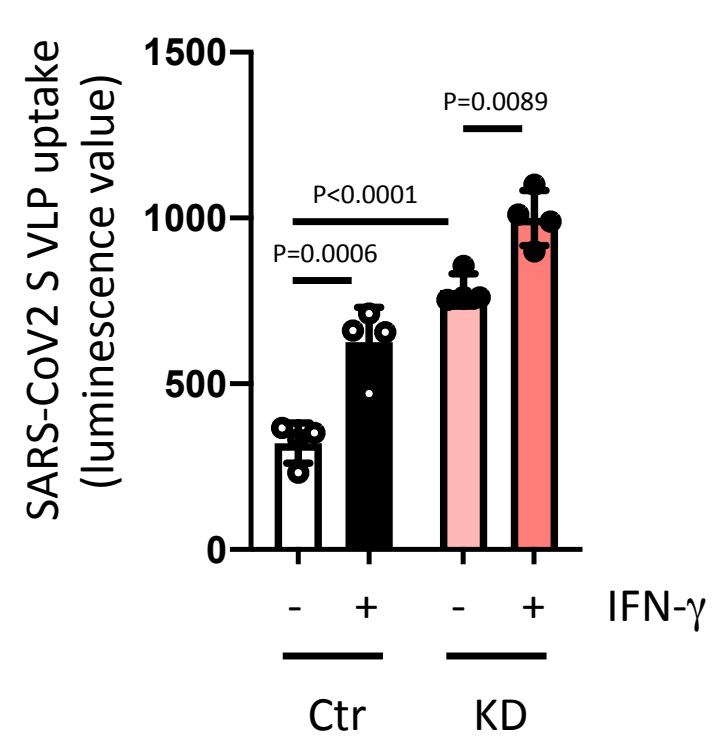

B

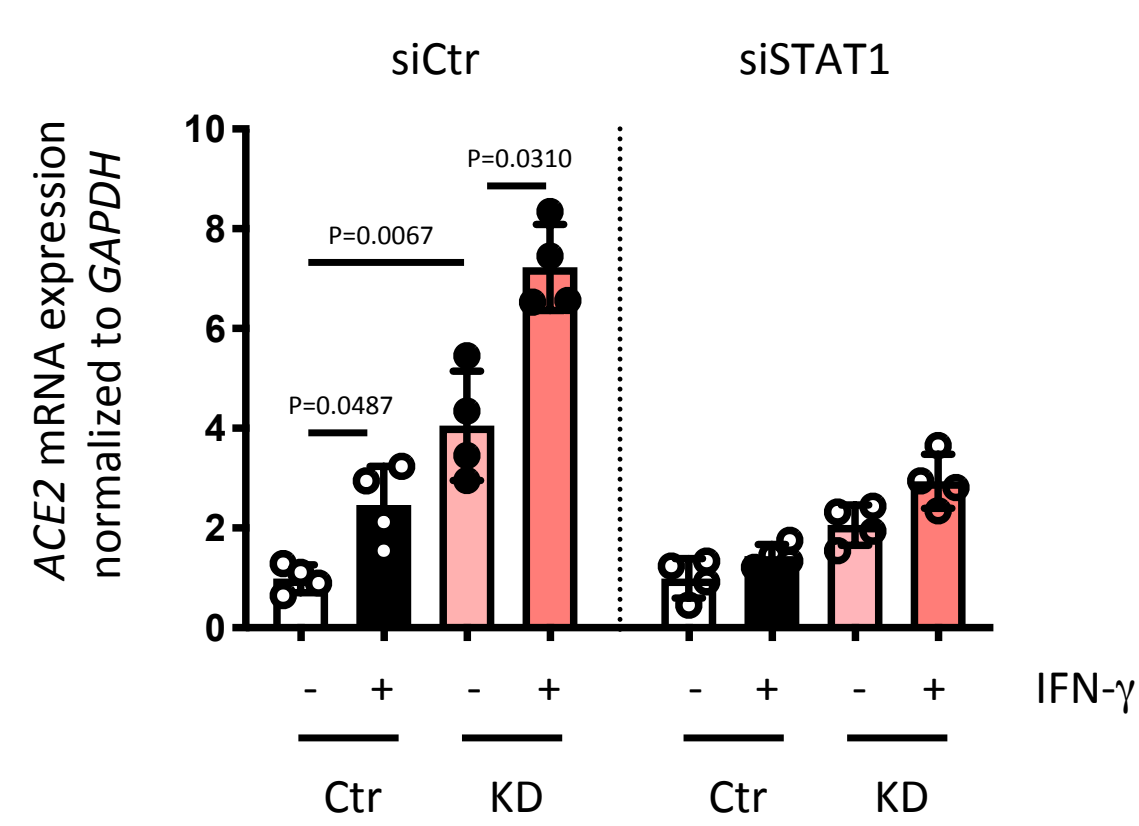

$\mathrm{E}$

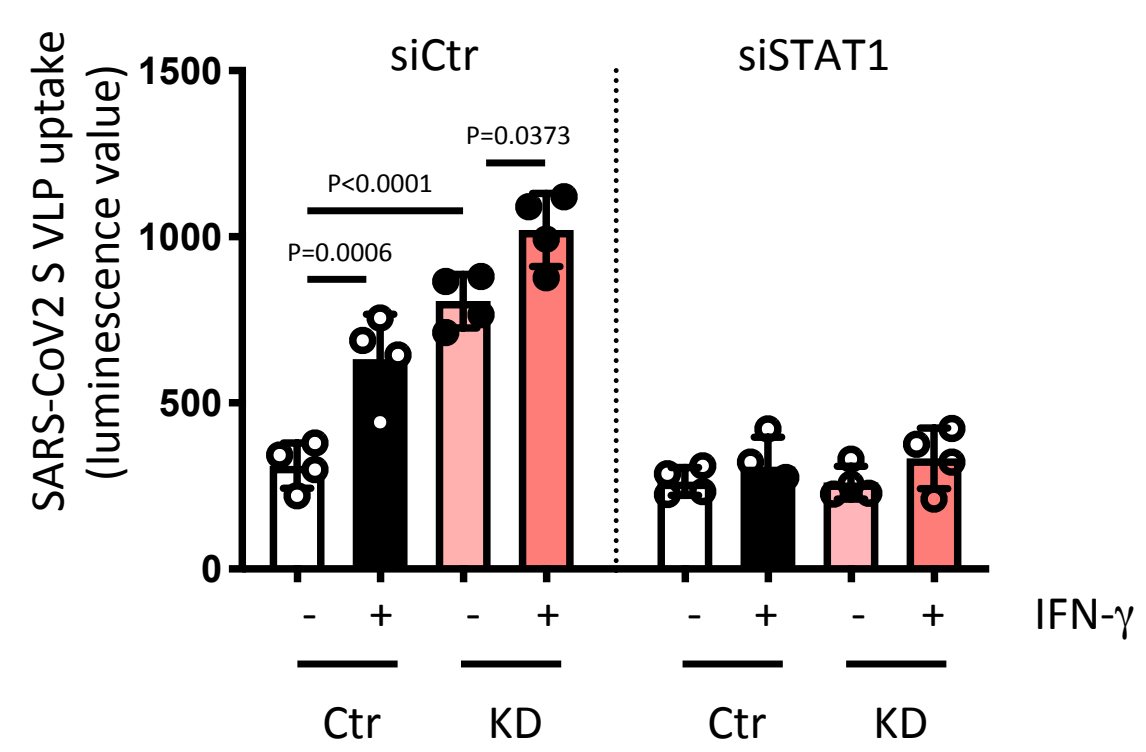

C Ctr siRNA STAT1 SIRNA

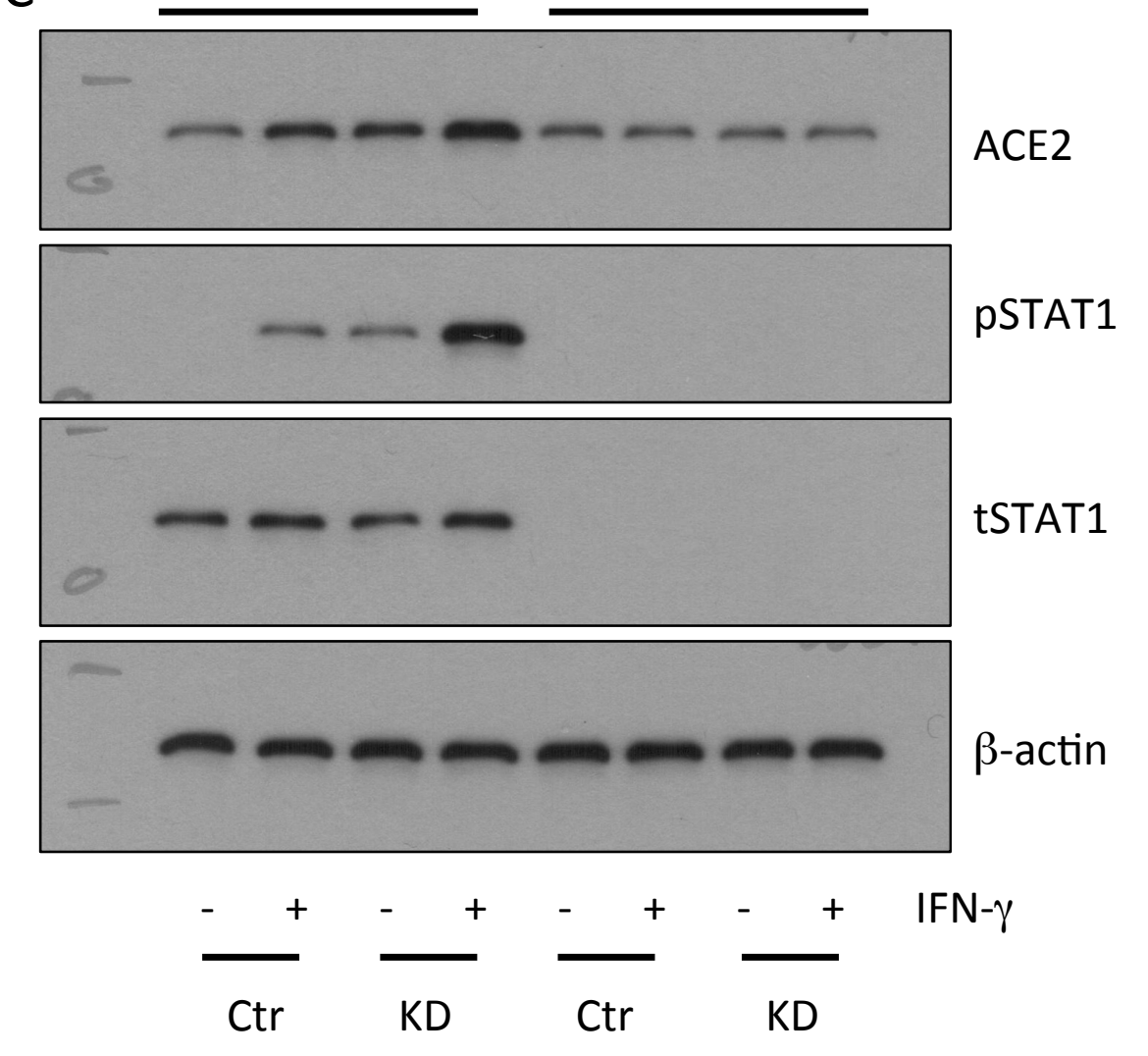

Figure 3. IFN- $\gamma$ promotes uptake of SARS-CoV2 spike-expressing VLPs in a STAT1-dependent manner. A) Caco-2BBe cells expressing non-targeting control (Ctr) or PTPN2-specific (KD) shRNA were treated with IFN- $\gamma$ for $24 \mathrm{~h}$ and analyzed for ACE2 mRNA expression. B) Ctr and KD Caco-2BBe cells were treated with non-targeting control (siCtr) or STAT1-specific (siSTAT1) siRNA prior to incubation with IFN- $\gamma$ for $24 \mathrm{~h}$ and analysis for ACE2 mRNA expression. C) Representative Western blot images for the indicated proteins from cells treated as in B. D) Ctr and KD Caco-2BBe cells were infected with VLPs expressing SARS-CoV-2 spike protein in the presence or absence of IFN- $\gamma$ and luminescence measured after $48 \mathrm{~h}$. E) Ctr and KD Caco-2BBe cells were treated with non-targeting control (siCtr) or STAT1-specific (siSTAT1) siRNA prior to infection with VLPs expressing SARS-CoV-2 spike protein in the presence or absence of IFN- $\gamma$ and luminescence measured after $48 \mathrm{~h}$. Statistical differences are indicated in the figure (One-way ANOVA, $n=4)$. Each dot represents the average of an independent experiment with 2-3 technical replicates, each. 
A

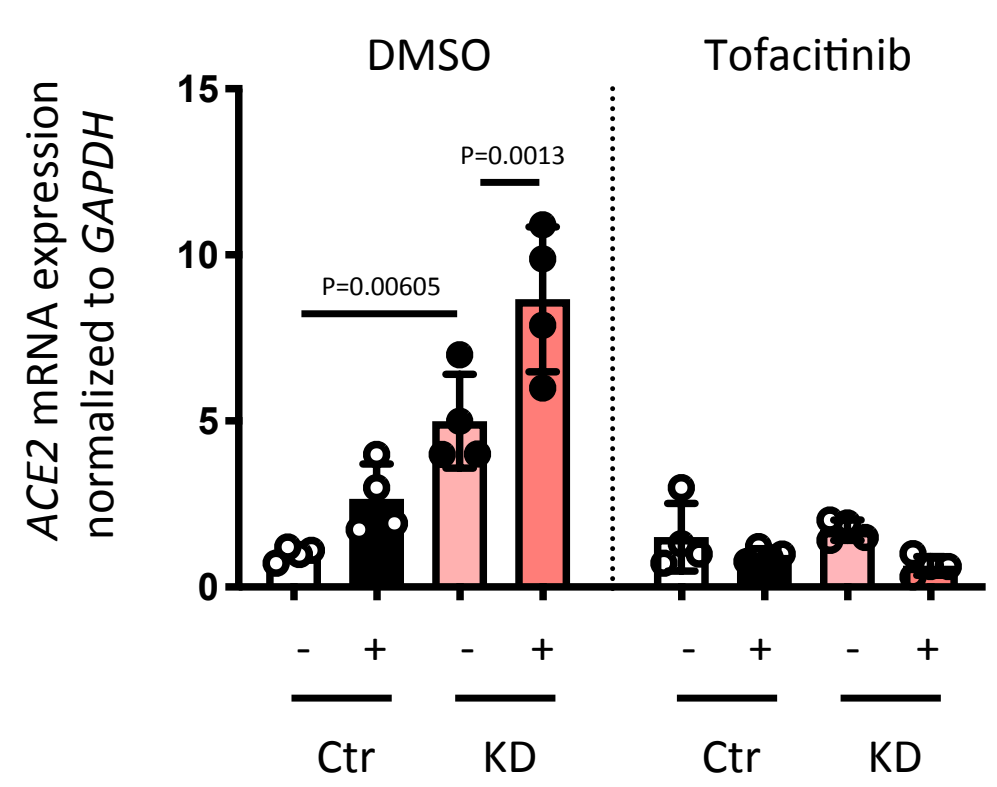

B

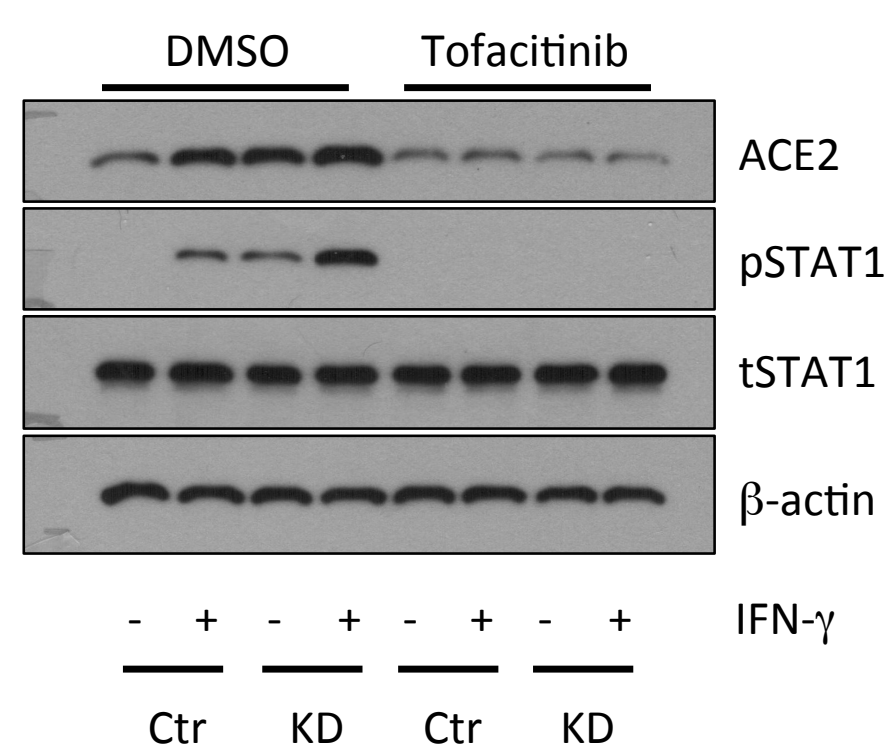

C

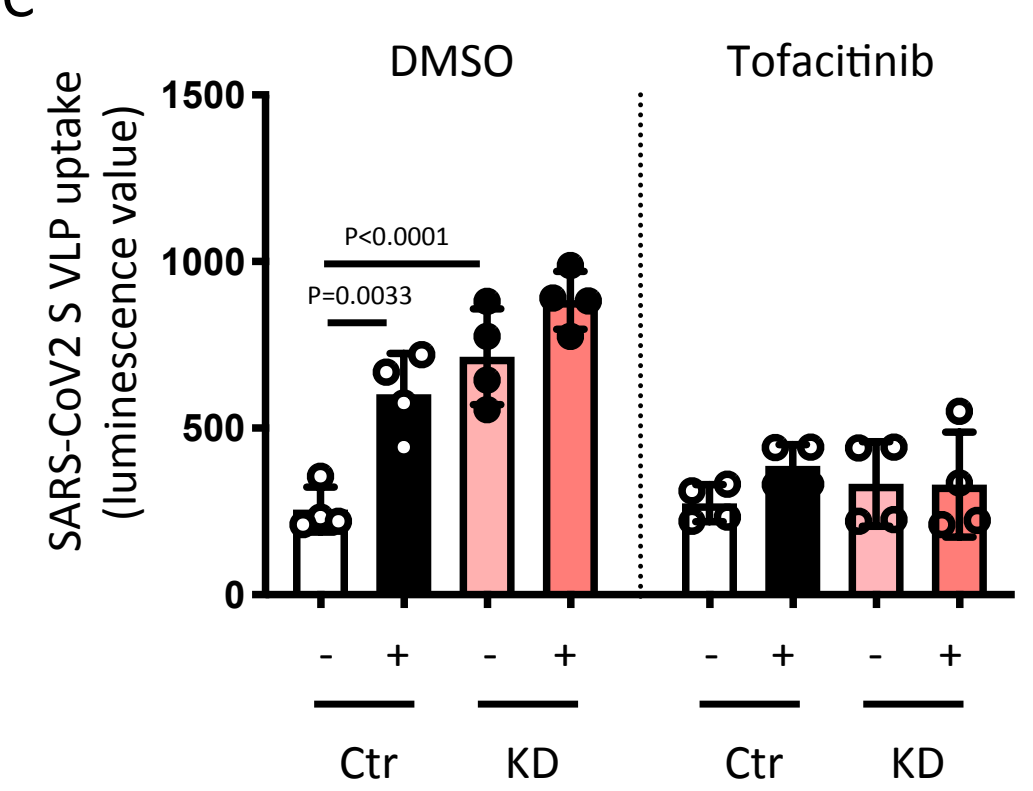

D

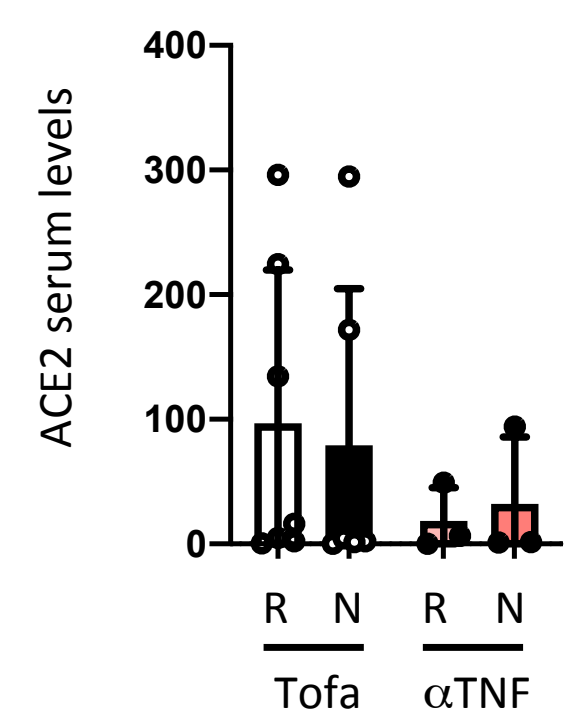

E

PBMC

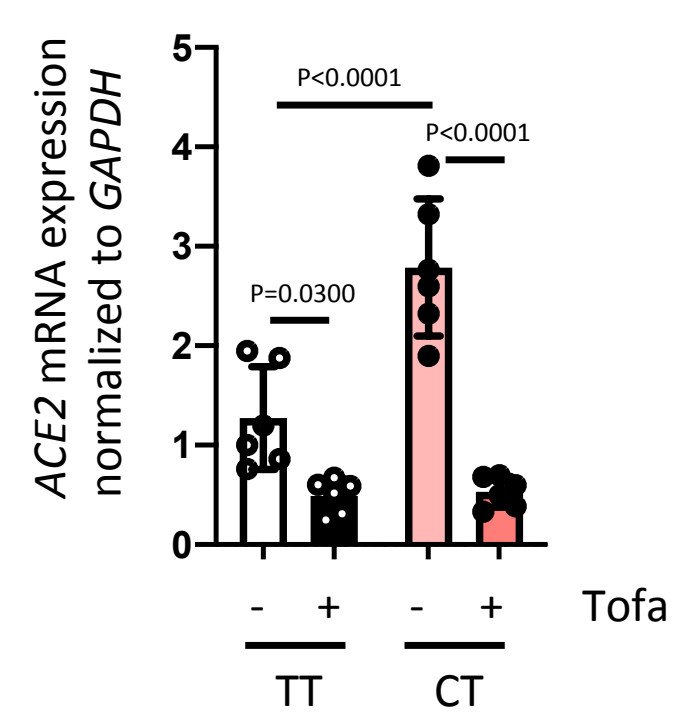

F

PBMC

G

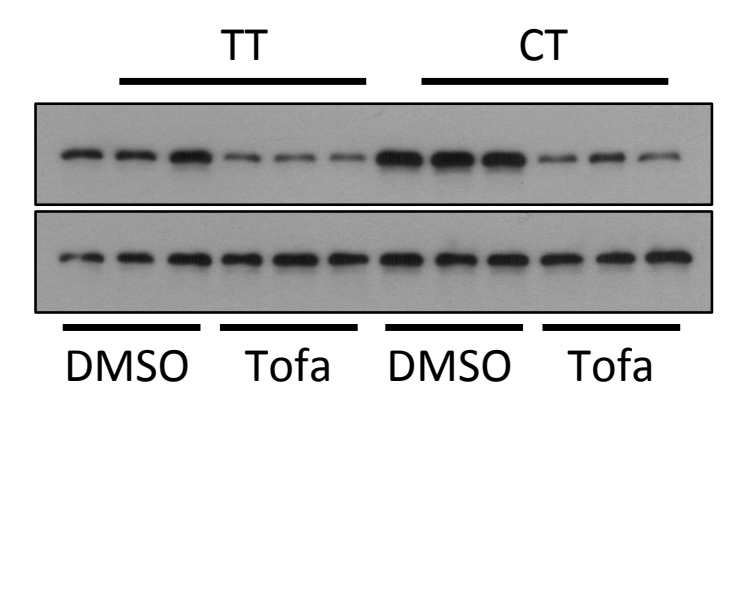

ACE2

$\beta$-actin

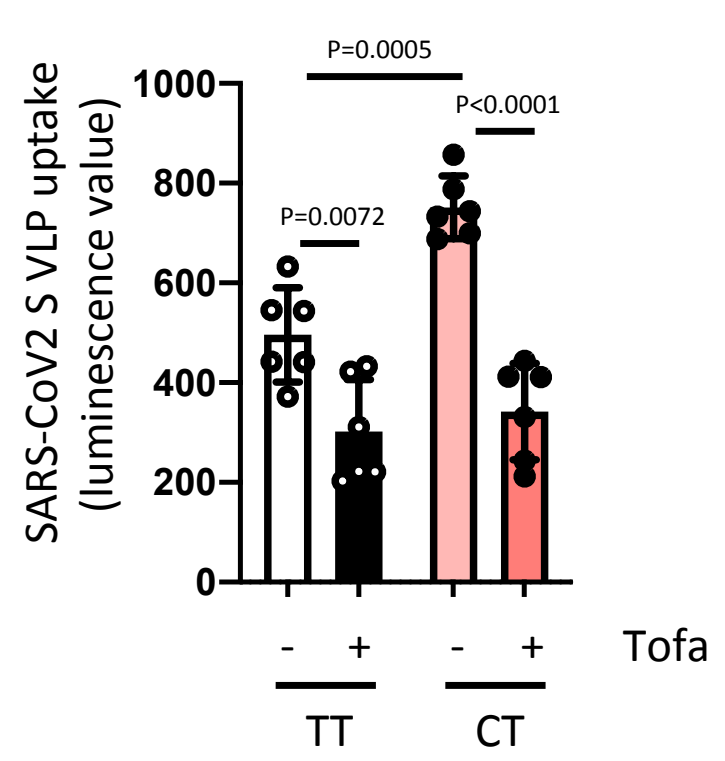

Figure 4. Tofacitinib prevents ACE2 upregulation and reduces SARS-CoV-2 VLP-uptake. A-C: Caco-2BBe cells expressing nontargeting control (Ctr) or PTPN2-specific (KD) shRNA were treated with vehicle (DMSO) or Tofacitinib for $1 \mathrm{~h}$ prior to infection with VLPs expressing SARS-CoV-2 spike protein in the presence or absence of IFN- $\gamma$. A) Relative mRNA expression of ACE2 and B) representative Western blot pictures for the indicated proteins $24 \mathrm{~h}$ after VLP treatment, C) luminescence values as an approximation of VLP uptake after 48 h. D) Serum from ulcerative colitis patients treated with tofacitinib or anti-TNF $\alpha$ were analyzed for ACE2 protein level ( $R=$ responder; NR = non-responder). E-G) Peripheral blood mononuclear cells from IBD patients homozygous for the major allele (TT) or heterozygous for the disease-associated risk allele in SNP rs1893217 in PTPN2 were treated with Vehicle (DMSO) or Tofacitinab for $24 \mathrm{~h}$ and analyzed for E) ACE2 mRNA and F) ACE2 protein expression. G) After $24 \mathrm{~h}$ Tofacitinib-treatment, the cells were infected with VLPs expressing SARS-CoV-2 spike protein and luminescence analyzed after $48 \mathrm{~h}$ as an approximation for VLP uptake. Statistical differences are indicated in the figure (One-way ANOVA, A-C: $n=4$; D: Tofacitinib treated patients $n=12$, anti-TNF-treated patients $n=6$ ); $E-G: n=6$ ). Each dot represents the average of an independent experiment (A-C) or a biological sample (D-G) with 2-3 technical replicates, each. 